\title{
Gravity as Entanglement Entanglement as gravity
}

(Introduction)

\author{
Vasil Penchev ${ }^{1}$
}

\section{The objectives are:}

- To investigate the conditions under which the mathematical formalisms of general relativity and of quantum mechanics go over each other.

- To interpret those conditions meaningfully and physically.

- To comment that interpretation mathematically and philosophically.

\section{Scientific prudence, or what are not our objectives:}

- To say whether entanglement and gravity are the same or they are not: For example, our argument may be glossed as a proof that any of the two mathematical formalisms needs perfection because gravity and entanglement really are not one and the same.

- To investigate whether other approaches for quantum gravity are consistent with that if any at all.

\section{Background:}

- Eric Verlinde's entropic theory of gravity (2009): "Gravity is explained as an entropic force caused by changes in the information associated with the positions of material bodies" (Verlinde 2009: 1).

- the accelerating number of publications on the links between gravity and entanglement, e.g. Jae-Weon Lee, Hyeong-Chan Kim, Jungjai Lee's "Gravity as Quantum Entanglement Force": "We conjecture that quantum entanglement of matter and vacuum in the universe tend to increase with time, like entropy and there is an effective force called quantum entanglement force associated with this tendency. It is also suggested that gravity and dark energy are types of the quantum entanglement force ..." (Lee, Kim, Lee 2010: 1). Or: Mark Van Raamsdonk's "Comments on quantum gravity and entanglement".

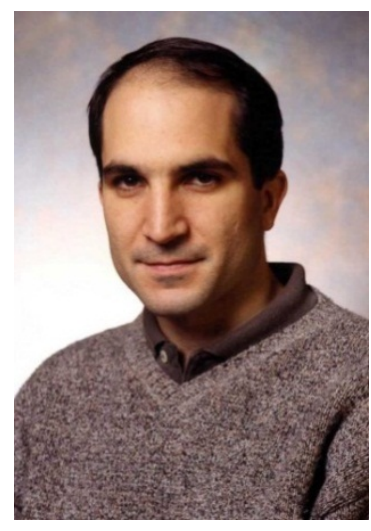

Figure 1: Dr. Juan Maldacena is the recipient of the prestigious Fundamental Physics Prize (3 million dollars)

- Juan Maldacena's "For the gauge/gravity duality" (Figure 1): "The gauge/gravity duality is an equality between two theories: On one side we have a quantum field theory in $d$ spacetime dimensions. On the other side we have a gravity theory on a $d+1$ dimensional spacetime that has an asymptotic boundary which is d dimensional" (Maldacena 2011: 1).

\footnotetext{
${ }^{1}$ Doctor of Science, Associate Professor in Dept.of Logical Systems and Models, Institute of Philosophy and Sociology, Bulgarian Academy of Sciences, vasildinev@gmail.com; http://vasil7penchev.wordpress.com ; http://www.scribd.com/vasil7penchev
} 


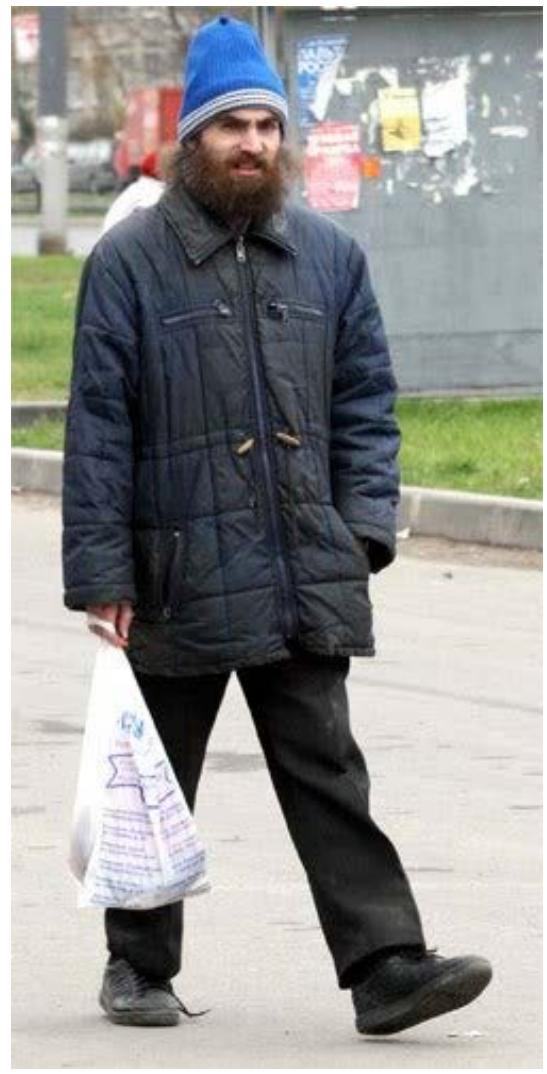

Figure 2: Gregory Perelman

- Poincaré conjecture: Every simply connected, closed 3-manifold is homeomorphic to the 3-sphere. Gregory Perelman (Figure 2) solved it (Perelman 2002; 2003; 2003), which is the third (of 7 and only solved) Millennium Prize Problem (the award refused). The corollary important is: 3D space is homeomorhic to a cyclic $3+1$ topological structure like the 3-sphere: e.g. the cyclically connected Minkowski space.

- The gauge/gravity duality \& Poincaré conjecture 3D (gauge) /3D+1 (gravity) are dual in a sense 3D \& a 3D+1 cyclic structure are homeomorphic "What about that duality, if $3 \mathrm{D}+1$ (gravity) is cyclic in a sense?" - will be one of our questions.

- The Higgs boson: It completes the standard model without gravity, even without leaving any room for it: The Higgs boson means: No quantum gravity! As the French academy declared "No perpetuum mobile" and it was a new principle of nature that generated thermodynamics: "No quantum gravity!" and it is a new very strange and amazing principle of nature! If the best minds tried a century to invent quantum gravity and they did not manage to do it, then it merely means that quantum gravity does not exist in principle. So that no sense in persisting to invent the "perpetuum mobile" of quantum gravity, however there is a great sense to build a new theory on that new principle:

1. The theory of gravity which is sure is general relativity, and it is not quantum: This is not a random fact.

2. If the standard model is completed by the Higgs boson but without gravity, then the cause for that is: The standard model is quantum. It cannot include gravity in principle just being a quantum theory.

3. Of course, a non-universality of quantum theory is a big surprise and quite incomprehensible at present, but all scientific experience of mankind is full of surprises.

General relativity vs. the standard model (Figure 3):

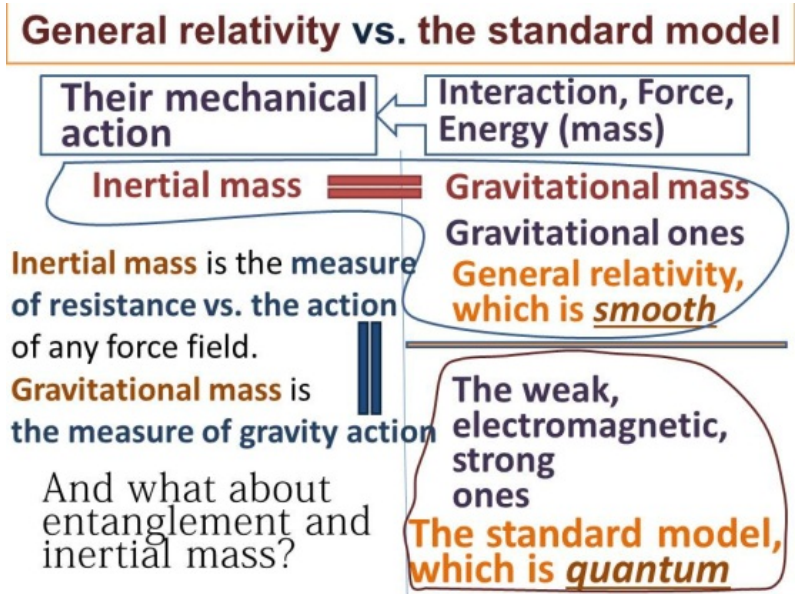

Figure 3 background is...

Our strategy on that

a) ... to show that entanglement is another and equivalent interpretation of the mathematical formalism of any force field (the right side of the previous slide);

b) ... to identify entanglement as inertial mass (the left side);

c) ... to identify entanglement just as gravitational mass by the equality of gravitational and inertial mass;

d) $\quad .$. to sense gravity as another and equivalent interpretation of any 
quantum-mechanical movement and in last analysis, of any mechanical (i.e. space-time) movement at all.

If we sense gravity as another and equivalent interpretation of any movement, then ... (figure 4):

- The standard model represents any quantum force field: strong, electromagnetic, or weak field.

- The standard model does not and cannot represent gravity because it is not a quantum at all: Instead of that, it is a smooth image of all quantum fields.
If we sense gravity as another and equivalent interpretation of any movement, then ...

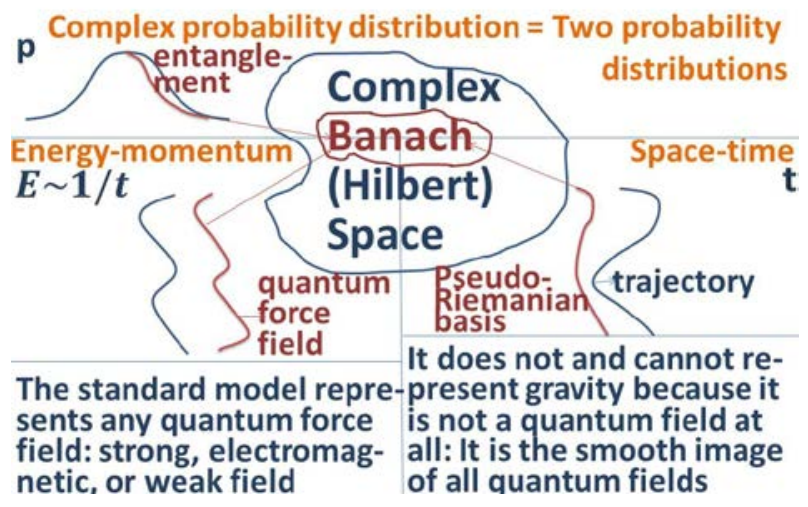

Figure 4

The Higgs boson is an answer ... and too many questions:

- What about the Higgs field? The standard model unifies electromagnetic, weak and strong field. Is there room for the Higgs field?

- What about the Higgs field and gravity?

- What about the Higgs field and entanglement?

- ... and too many others ...

We will consider the Higgs field as a "translation" of gravity \& entanglement in the language of the standard model as a theory of unified quantum field. However what does "quantum field" mean? Is not this a very strange and controversial term?

Quantum field means that field whose value in any space-time point is a wave function. If the corresponding operator between any two field points is self-adjoint, then:

- A quantum physical quantity corresponds to it, and:

- All wave function and selfadjoint operators share a common Hilbert space or in other words, they are not entangled.

Quantum field is the only possible field in quantum mechanics, because:

- It is the only kind of field which can satisfy Heisenberg's uncertainty.

- The gradient between any two field points is the gradient of a certain physical quantity.

However the notion of quantum field does not include or even maybe excludes that of entanglement: If our suspicion about the close connection between entanglement and gravity is justified, then this would explain the difficulties about “quantum gravity”. Then we can outline the path to gravity from the viewpoint of quantum mechanics - as an appropriate generalization of 'quantum field' so that to include 'entanglement'. If all wave functions and operators (which will not already be selfadjoint in general) of the quantum field share rather a common Banach than Hilbert space, this is enough. That quantum field is a generalized one.

However there would be some troubles with its physical interpretation. Which are the troubles and rather the "cure" for them? The "cure" for them is to be generalized correspondingly the notion of quantity in quantum mechanics. If the operator is in Banach space (correspondingly, yet no selfadjoint operator), then its functional is a complex number in general. Its modulus is the value of the physical quantity. The expectation of two quantities is nonadditive in general. The quantity of subadditivity (which can be zero, too) is the degree (or quantity) of entanglement, $e$ : 


$$
e=\sqrt{A_{1}+A_{2}} \text { in: } A_{1}+A_{2}-\sqrt{A_{1}+A_{2}},
$$

where $A_{1}, A_{2}$ are as quantities in the two entangled quantum systems 1 and 2 . To recall that any quantity $A$ in quantum mechanics is defined as mathematical expectation, i.e. as a sum or integral of the product of any possible value and its probability, or as a functional:

$$
\mathrm{A}=\int_{-\infty}^{\infty} a \mathrm{p}_{\mathrm{a}} \mathrm{d} a=\int_{-\infty}^{\infty} \Psi \widehat{\mathrm{A}}\left(\Psi^{*}\right)
$$

$e$ cannot be quantized in principle even if $A_{1}, A_{2}$ are quantum or quantized, because as expectation as probability are neither quantum, nor quantizable since wave function is smooth (a "leap” in probability would mean infinite energy)

Granted entanglement and gravity are the same or closely connected, this explains:

(1) why gravity cannot be quantized;

(2) why gravity is always nonnegative (there is no antigravity).

\section{Then what is gravity?}

It cannot be define in terms of "classical" quantum field, but only in those of generalized quantum field. It is always the smooth curvature or distortion of "classical" quantum field. It is an interaction (force, field) of second order: rather the change of quantum field in space-time than a new quantum field. That change of quantum field is neither quantum, nor quantizable: It cannot be a new quantum field in principle. Its representation as a whole (or from the "viewpoint of eternity") is entanglement. Then, in a few words, what would gravity be in terms of generalized quantum field? The answer is: a smooth space-time DoF constraint imposed on any quantum entity by any or all others. Entanglement is another (possibly equivalent) mapping of gravity from the probabilistic rather than space-time viewpoint of "eternity". The smooth space-time DoF constraint in each moment represents a deformed "inwards"3D light sphere of the 4-Minkowski-space light cone ("outwards" would mean antigravity). The wellordered (in time) set of all such spheres in all moments constitutes the pseudo-Riemannian space of general relativity.

The conception of "second quantization" in the language of quantum field theory: What does the "second quantization" mean in terms of the "first quantization"? If the "first quantization" gives us the wave function of all the quantum system as a whole, then the "second quantization" divides it into the quantum subsystems of "particles" with wave functions orthogonal between each other; or in other words, these wave functions are not entangled. Consequently, the "second quantization" excludes as entanglement as gravity in principle.

The second quantization in terms of Hilbert space: The second quantization divides infinitedimensional Hilbert space into also infinitedimensional subspaces. A subspace can be created or annihilated: This means that a particle is created or annihilated. The second quantization juxtaposes a certain set of Hilbert subspaces with any space-time point. One or more particles can be created or annihilated from any point to any point. However though the Hilbert space is divided into subspaces from a space-time point to another in different ways, all subspaces share it.

A philosophical interpretation both of quantum (I) and of quantized (II) field: Quantum v quantized field means for any space-time point to juxtapose the Hilbert space and one division into subspaces of its. 
The gauge theories interpret that as if the Hilbert space with its division into subspaces is inserted within the corresponding space-time point. Any quantum conservation law is a symmetry or a representation into Hilbert space of the corresponding group. The standard model describes the general and complete group including all the "strong”, "electromagnetic" and "weak" symmetries

A philosophical interpretation as to the closedness of the standard model: The standard model describes the general and complete group including all the "strong", "electromagnetic" and "weak" symmetries within any space-time point. Consequently the standard model is inside of any space-time point, and describes movement as a change of the inside structure between any two or more space-time points. However gravity is outside and remains outside of the standard model: It is a relation between two or more space-time points but outside and outside of them as wholenesses. There is need to add an interpretation of quantum duality à la Nicolas of Cusa: After Niels Bohr quantum duality has been illustrated by the Chinese Yin and Yang, which will be now juxtaposed in scale in Nicolas of Cusa's manner:

- Yin becomes Yang as the smallest becoming the biggest, and vice versa:

- Yang becomes Yin as the biggest becoming the smallest.

- Besides moreover, Yin and Yang continue to be as parallel as successive in the same

scale.

And now, from the philosophical to the mathematical and physical ...: (figure 5):

And now, from the philosophical to the mathematical and physical ....

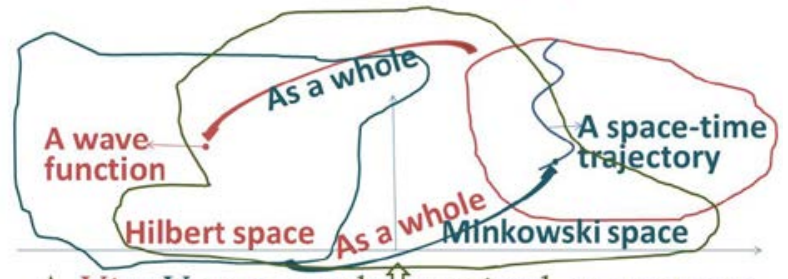

A Yin-Yang mathêmatical structure

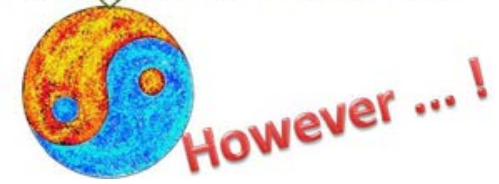

Figure 5
Its essence is to be juxtaposed cyclically both pairs:

The first one is the pair of wave function and world line, and the second one is that of their spaces: correspondingly Hilbert and Minkowski space.

The Hilbert space turns out to be inserted in in any separate point of the Minkowski space, but the latter is inserted back cyclically in any separate point of the former, too.

The basic idea of the gauge theories is complemented by its mirror twin completing it cyclically and fractally into a perfect symmetry.

However ...: Have already added à la Nicolas of Cusa's interpretation to that

Yin-Yang structure, so that ... (figure 6): In last analysis we got a cyclic and fractal YinYang mathematical structure ... Will check whether it satisfies our requirements:

- Yin and Yang are parallel to each other.

- Yin and Yang are successive to each other.

- Yin and Yang as the biggest are within themselves as the smallest.
However ...: Have already added à la

Nicolas of Cusa's interpretation to that Yin-Yang structure, so that ...

The "biggest" of the space-time whole is inserted within

the "smallest" of any space-time point The "biggest" of the
Hilbert-space whole
is inserted within
the "smallest" of any Hilbert-space point

Figure 6 


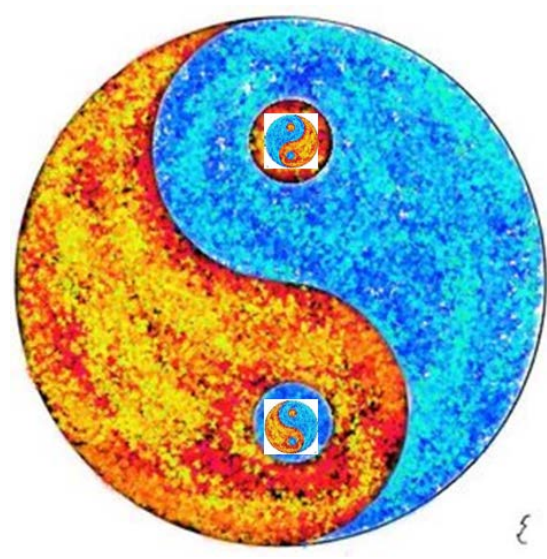

space-time and vice versa.

Besides, please note: it being cyclic need not be infinite! Need only two entities, "Yin and Yang", and a special structure tried to be described above.

Will interpret that Yin-Yang structure in terms of the standard model \& gravity. Our question is how the gravity being "outside" space-time points as a curving of a smooth trajectory, to which they belong, will express itself inside, i.e. within space-time points representing Hilbert space divided into subspaces in different ways. Will try to show that: The expression of gravity "outside" looks like entanglement "inside" and vice versa. Besides, the expression of entanglement "outside" looks like gravity inside of all the

Back to the philosophical interpretation of quantum (I) or quantized (II) field: The principle is: The global change of a space-time trajectory (or an operator in pseudoRiemannian space) is equivalent to, or merely another representation of a mapping between two local Hilbert spaces of Banach space (entanglement). The same principle from the viewpoint of quantum mechanics and information looks like as follows: Entanglement in the "smallest" returns and comes from the "outsides" of the universe, i.e. from the "biggest”, as gravity.

Back to the philosophical interpretation, or more and more „miracles“: Turns out the yet "innocent" quantum duality generates more and more already "vicious" dualities more and more extraordinary from each to other, namely those of:

... the continuous (smooth) \& discrete;

... whole \& part;

... the single one \& many;

... eternity \& time;

... the biggest \& smallest;

... the external \& internal;

... and even ... of " $\&$ " and duality.

Where "\&” means:

... equivalence;

... relativity;

... invariance;

... conservation.

The second quantization in terms of Banach space: If the Banach space is smooth, it is locally "flat", which means that any point of it separately implies a "flat" and "tangential" Hilbert space at this point. However the system of two or more points in Banach space does not share in general a common tangential Hilbert space, which is another formulation of entanglement. One can always determine a self-adjoint operator (i.e. a physical quantity) between any two points in Banach space (i.e. between the two corresponding tangential Hilbert spaces mapping by the operator).

If we can always determine a self-adjoint operator (i.e. a physical quantity) between any two points in Banach space, then follows the second quantization is invariant (or the same) from Hilbert to any smooth Banach space, and vice versa, consequently between any two smooth Banach spaces. 
As entanglement as gravity is only external, or both are "orthogonal” to the second quantization: It means that no interaction or unity between both gravity and entanglement, on the one hand, and the three rest, on the other, since the latters are within Hilbert space while the formers are between two (tangential) Hilbert spaces. However as entanglement as gravity can be

\section{The problem of Lorentz invariance Try to unite the following facts: \\ The Lorentz noninvariant are:}

\begin{tabular}{|c|c|}
\hline Newton's mechanics & $\begin{array}{l}\text { Schrödinger's } \\
\text { quaantum mechanics }\end{array}$ \\
\hline \multicolumn{2}{|c|}{ The Lorentz invariant are: } \\
\hline $\begin{array}{l}\text { Maxwell's theory of } \\
\text { electromagnetic field } \\
\text { Einstein's special relativity }\end{array}$ & $\begin{array}{l}\text { Dirac's } \\
\text { quantum mechanics } \\
\text { of electromagnetic field }\end{array}$ \\
\hline \multicolumn{2}{|c|}{$\begin{array}{l}\text { The locally Lorentz invariant } \\
\text { (but noninvariant globally) are: }\end{array}$} \\
\hline Einstein's general relativity & $\begin{array}{l}\text { Our hypothesis } \\
\text { of entanglement \& } \\
\text { gravity }\end{array}$ \\
\hline Relativity & Quantum theory \\
\hline
\end{tabular}

Figure 7 divided into the second-quantized parts (subspaces) of the Hilbert space, which "internally" is granted for the same though they are at some generalized "angle" "externally".

The problem of Lorentz invariance (figure 7): ... whether gravity is not a "defect" of electromagnetic field... However mass unlike electric (or Dirac's magnetic) charge is a universal physical quantity which characterizes anything existing. A perfect, “Yin-Yang” symmetry would require as the locally "flat" to become globally "curved" as the locally "curved" to become globally "flat" as the "biggest" to return back as the smallest and locally "flat".

For example this might mean that the universe would have a charge (perhaps Dirac's "monopole" of magnetic charge), but not any mass: the curved Banach space can be seen as a space of entangled spinors.

Electromagnetic field as a "Janus" with a global and a local "face": Such a kind of consideration like that in the previous slide cannot be generalized to the "weak" and "strong" field: They are always local since their quanta have a nonzero mass at rest unlike the quantum of electromagnetic field: the photon. As to the electromagnetic field, both global and local (the latter is within the standard model) consideration is possible.

Gravity (\& entanglement) is only global (external), weak \& strong interaction is only local (internal), and electromagnetic field is both local and global: It serves to mediate both between the global and the local and between the external and the internal. Consequently, it conserves the unity of the universe.

More about the photon two faces:

- It being global has no mass at rest.

- It being local has a finite speed in spacetime.

In comparison with it:

- Entanglement \& gravity being only global has no quantum, thus neither mass at rest nor a finite speed in spacetime.

- Weak \& strong interaction being only local has quanta both with a nonzero mass at rest and with a finite speed in spacetime.

Lorentz invariance has a local and a global face, too: In turn, this generates the two faces of photon. The local "face" of Lorentz invariance is both within and at any spacetime point. It "within" such a point is as the "flat" Hilbert space, and "at" it is as the tangential, also "flat" Minkowski space. 
Its global "face" is both "within" and "at" the totality of the universe. It is "within" the totality flattening Banach space by the axiom of choice. It is "at" the totality transforming it into a spacetime point

It is about time to gaze that Janus in details in Dirac's brilliant solving by spinors:

In terms of philosophy, "spinor" is the total half (or "squire root") of the totality. In terms of physics, it generalizes the decomposition of electromagnetic field into its electric and magnetic component. The electromagnetic wave looks like the following:

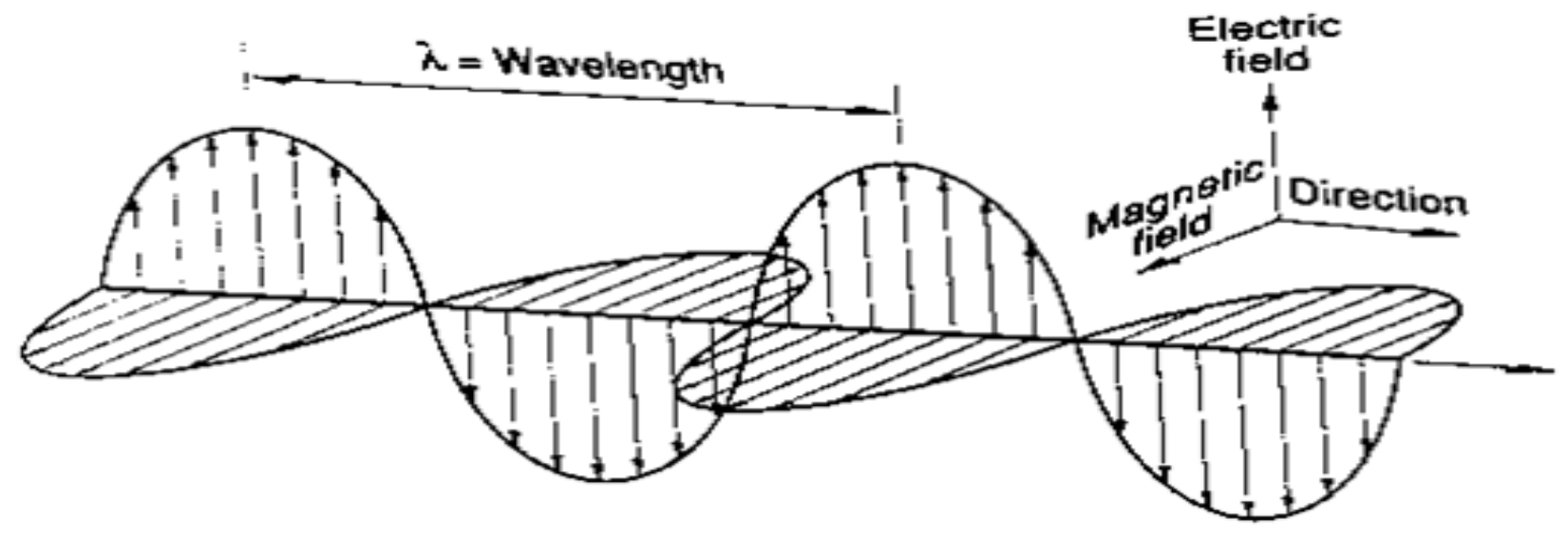

Figure 8

That is a quantum kind of generalization. Why? First, the decomposition into a magnetic and an electric component is not a decomposition of two spinors because the electromagnetic field is the vector rather than tensor product of them. Both components are exactly defined in any point time just as position and momentum as to a classical mechanical movement. The quantity of action is just the same way the vector than tensor product of them. Consequently, there is another way (the Dirac one) quantization to be described: as a transition or generalization from vector to tensor product.

Well, what about such a way gravity to be quantized? The answer is really quite too surprising: General relativity has already quantized gravity this way! That is general relativity has already been a quantum theory and that is the reason not to be able to be quantized once again just as the quant itself cannot be quantized once again!

What only need is to gaze at it and contemplate it to see how it has already sneaked to become a quantum theory unwittingly:

Cannot be, or general relativity as a quantum theory: Of course the Dirac way of keeping Lorentz invariance onto the quantum theory is the most obvious for general relativity: It arises to keep and generalize just the Lorentz invariance for any reference frame. However the notion of reference frame conserves the smoothness of any admissable movement requiring a definite speed toward any other reference frame or movement. Should see how the Dirac approach generalizes implicitly and unwittingly "reference frame" for discrete (quantum) movements. How? 
"Reference frame" after the Dirac approach: "Reference frame" is usually understood as two coordinate frames moving to each other with a relative speed, $v(t)$. However we should already think of it after Dirac as the tensor product of the given coordinate frames. This means to replace $v(t)$ with $\delta(t)$ (Dirac delta function) in any $t=t_{0}$.

Given a sphere $S$ with radius, $\sqrt{x^{2}+y^{2}+z^{2}+|v|^{2} t^{2}}$, it can represent any corresponding reference frame in Minkowski space. $\boldsymbol{S}$ can be decomposed into any two great circles $\boldsymbol{S}_{\mathbf{1}} \otimes \boldsymbol{S}_{\mathbf{2}}$ of its, perpendicular to each other, as the tensor product $\otimes$ of them. Given a sphere $S$ with radius $\sqrt{x^{2}+y^{2}+z^{2}+|v|^{2} t^{2}}$ decomposed into any two great circles, $S_{1} \otimes S_{2}$, and $\boldsymbol{S}, \boldsymbol{S}_{1}, \boldsymbol{S}_{\mathbf{2}}$ are with the same radius. We can think of $\boldsymbol{S}_{1}, \boldsymbol{S}_{2}$ as the two spinors of a reference frame after Dirac

If we are thinking of Minkowski space as an expanding sphere, then its spinor decomposition would represent two planar, expanding circles perpendicular to each other, e.g. the magnetic and electric component of electromagnetic wave as if being quantumly independent of each other.

The praising and celebration of sphere: The well-known and most ordinary sphere is the crosspoint of:

... quantization

... Lorentz invariance

... Minkowski space

... Hilbert space

... qubit

... spinor decomposition

... electromagnetic wave

... wave function

possible!

... making their uniting, common consideration, and mutual conceptual translation -

More about the virtues of the sphere: It is the "atom" of Fourier transform:

The essence of Fourier transform is the (mutual) replacement between the argument of a function and its reciprocal:

or „quantumly“:

$$
f(t) \leftrightarrow f\left(\frac{1}{t}\right)=f(\omega),
$$

(Equation 3)

As such an atom, it is both:

$$
f(t) \leftrightarrow f(E) .
$$

(Equation 4)

- as any harmonic in Hilbert space:

$$
f_{n}(\omega)=e^{i n \omega}
$$

(Equation 5)

- as any inertial reference frame in Minkowski space:

$$
f(t)=r(t)=\sqrt{c^{2} t^{2}-x^{2}-y^{2}-z^{2}} .
$$

(Equation 6)

Again about the spinor decomposition: Since the sphere is what is "spinorly" decomposed into two orthogonal great circles, the spinor decomposition is invariant to Fourier transform or to the mutual transition of Hilbert and Minkowski space. In particular this implies the spinor decompsition of wave function and even of its "probabilistic interpretation": Each of its two real "spinor" components can be interpreted as the probability both of a discrete quantum leap to, and of a smooth reaching the corresponding value. 
A necessary elucidation of the connection between probabilistic (mathematical) and mechanical (physical) approach (figure 9):

A necessary elucidation of the connection

between probabilistic (mathematical) and mechanical (physical) approach

No axiom of choice (the Paradise)

Probabilistic (mathematical) approach Totality aka eternity aka infinity

Mechanical (physical) approach

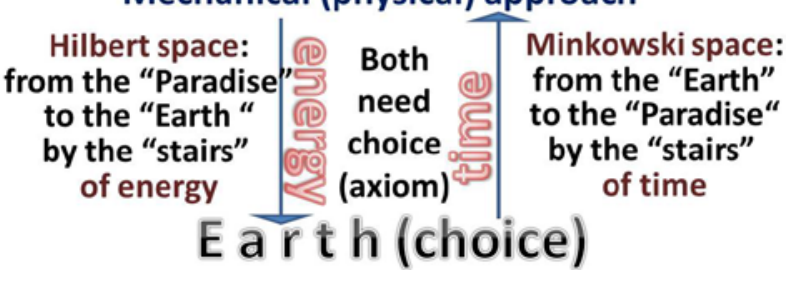

Figure 9
The mathematical approach and the physical one approach are very closely and explicitly connected in quantum mechanics: There is no boundary, but a link between them. The mathematical axiom of choice and the physical electromagnetic wave play the role of that link:

All the space-time is well-ordered thus it requires the axiom of choice or the electromagnetic wave, which can implement that.

The physical meaning of the Poincare conjecture (proved by Perelman) is that the $3 \mathrm{D}$ Euclidean space is equivalent to the light in vacuum, i.e. they are the same. The corollaries are rather interesting: For example, the light is what creates the 3D Euclidean space (well-)ordering it. Or: as the Poincare conjecture, as the axiom of choice is embedded in the light as the boundary, the connection and the link between the mathematical and the physical.

Coherent state, statistical ensemble, and two kinds of quantum statistics: The process of measuring transforms the coherent state into a classical statistical ensemble. Consequently, it requires the axiom of choice. However yet the mathematical formalism of Hilbert space allows two materially different interpretations corresponding to the two basic kinds of quantum statistics, of quantum indistinguishability, and of quantum particles: bosons and fermions:

The axiom of choice as the boundary between bosons and fermions: The two interpretations of a coherent state mentioned above are:

- As a nonordered ensemble of complex (= two real ones) probability distribution after missing the axiom of choice: aka bosons.

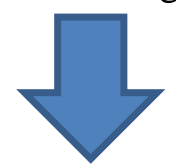

\section{The axiom of choice}

- As a well-ordered series either in time or in frequency (energy) equivalent to the axiom of choice: aka fermions.

The sense of quantum movement represented in Hilbert space: The way of generalization from classical to quantum movement is as follows:

- A common (namely Euclidean) space includes the two aspects of any classical movement, which are static and dynamic one and corresponding physical quantities to each of them.

- Analogically, a common (namely Hilbert) space includes the two aspects of any quantum movement: static (fermion) and dynamic (boson) one, and their physical quantities.

- Quantum vs. classical movement: However the two (as static as dynamic) aspects of classical movement are included within the just static (fermion) aspect of quantum movement as the two possible "hypostases" of the same quantum state. 
The static (fermion) aspect of quantum movement shows at a quantum leap (the one fermion of the pair) or at the equivalent smooth trajectory between the same states (the other). These two fermions for the same quantum state can be seen as two spinors keeping Lorentz invariance.

The spin statistics theorem about fermions: If one swaps the places of any two quantum particles, this means to swap the places between "particle" and "field", or in other words to reverse the direction "from time to energy" into "from energy to time", or to reverse the sign of wave function.

The following set-theory explanation may be useful: If there are many things, which are the same or "quantumly indistinguishable", there are anyway two opportunities: either to be "well-ordered" as the positive integers are (fermions), or not to be ordered at all as the elements of a set (bosons). Though indistinguishable, the swap of their corresponding ordinal (serial) number is distinguishable in the former case unlike the latter one.

\section{Positive integers vs. fermions vs. bosons illustrated (figure 10):}

However yet that "positive-integers analogy” is limited:

The well-ordering of positive integers has "memory" in a sense: One can distinguish two swaps, too, rather than only being odd or even number of swaps available (as fermions).

The well-ordering of fermions has no such memory. The axiom of choice and wellordering theorem do not require such a memory.

However if all the choices (or the choices after the well-ordering of a given set) constitute a set, then such a memory is posited just by the axiom of choice.

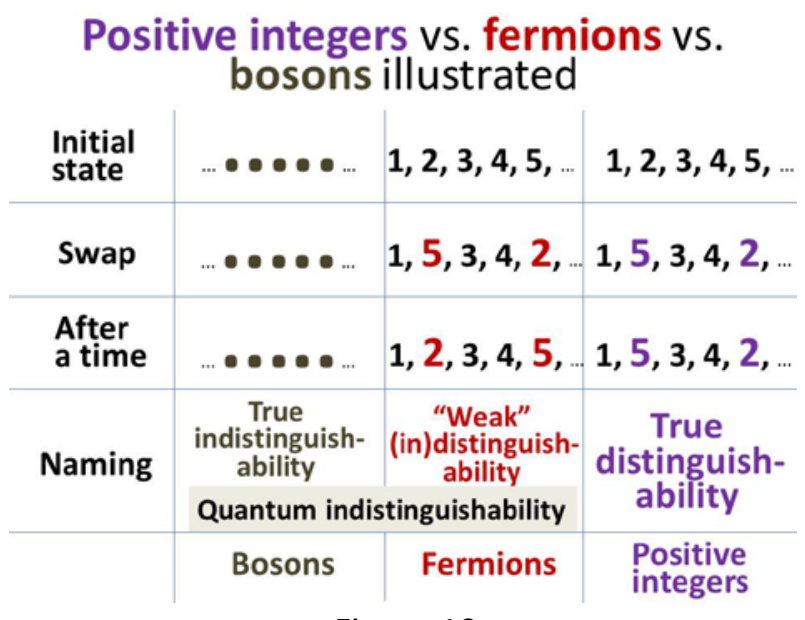

Figure 10 (figure 11):

\section{Quantum vs. classical movement in terms of (quantum in)distinguishability}

Quantum vs. classical movement in terms of (quantum in)distipguishability

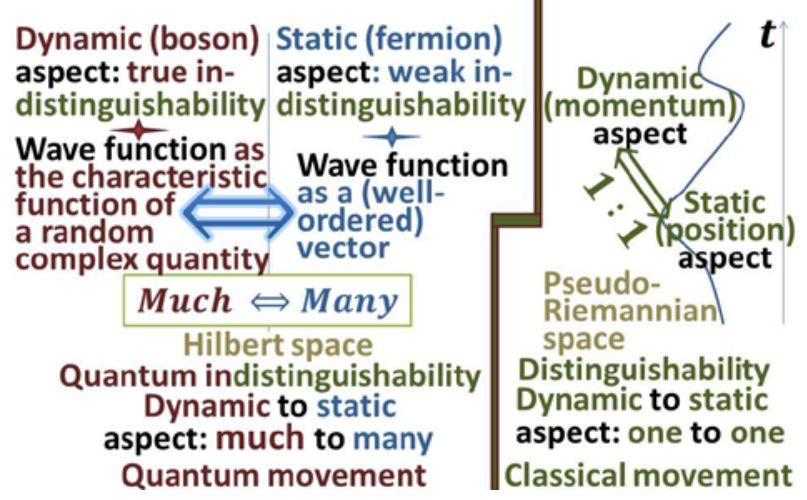

Figure 11
Our interpretation of fermion antisymmetry vs. boson symmetry follows:

The usual interpretation suggests that both the fermion and boson ensembles are wellordered: However any fermion swap reverses the sign of their common wave function unlike any boson swap.

Our interpretation is quite different: Any ensemble of bosons is not and cannot be wellordered in principle unlike a fermion one: The former is "much" rather than "many", which is correct only as to the latter. 
The well-ordering of the unorderable - fermions vs. bosons: The unorderable boson ensemble represents the real essence of quantum field unlike the "second quantization". The latter replaces the former almost equivalently with a well-ordered, as if a "fermion" image of it. In turn this hides the essence of quantum movement, which is "much - many", substituting it with a semi-classical "many - many".

\section{What will "spin" be in our interpretation?}

In particular, a new, specifically quantum quantity, namely "spin", is added to distinguish between the well-ordered (fermion) and the unorderable (boson) state in a wellordered way. However this makes any quantum understanding of gravity (or so-called "quantum gravity") impossible, because "quantum" gravity requires the spin to be an arbitrary real number In other words, gravity is the process in time (i.e. the time image of that process), which wellorders the unorderable The true "much - many" transition permits as a "many" (gravity in time, or "fermion") interpretation as a "much" (entanglement out of time, or "boson") interpretation.

Our interpretation of fermion vs. boson wave function: In turn it requires distinguishing between:

- the standard, "fermion" interpretation of wave function as a vector in Hilbert space (a square integrable function), and

- a new, "boson"interpretation of it as the characteristic function of a random complex quantity.

The former represents the static aspect of quantum movement, the latter the dynamic one. The static aspect of quantum movement comprises both the static (position) and dynamic (momentum) aspect of classical movement, because both are well-ordered, and they constitute a common well-ordering.

Entangled observables in terms of "spin" distinction: The standard definition of quantum quantity as "observable" allows its understanding:

- as a "fermion - fermion" transform;

- as a "boson - boson" one;

- as well as "fermion - boson" and "boson - fermion" one.

Only entanglement and gravity can create distinctions between the former two and the latter two cases. Those distinctions are recognizable only in Banach space, but vanishing in Hilbert space

The two parallel phases of quantum movement: Quantum field (the bosons) can be thought of as the one phase of quantum movement parallel to the other of fermion well-ordering: The phase of quantum field requires the universe to be consider as a whole or indivisible "much" or even as a single quant. The parallel phase of well-ordering (usually represented as some space, e.g. space-time) requires the universe to yield the well-known appearance of immense and unbounded space, cosmos, i.e. of an indefinitely divisible "many" or merely as many quanta.

\section{Why be "quantum gravity" a problem of philosophy rather than of physics?}

The Chinese Taiji 太極 (literally "great pole"), the "Supreme Ultimate", can comprise both phases of quantum movement. Then entanglement \& gravity can be seen as Wuji 無極 "Without Ultimate". 
In other words, gravity can be seen as quantum gravity only from the "Great Pole". This shows why "quantum gravity" is rather a problem of philosophy than (and only then) a such one of physics.

Hilbert vs. pseudo-Riemannian space - a preliminary comparison: As classical as quantum movement need a common space uniting the dynamic and static aspect: Hilbert space does it for quantum movement, and pseudo-Riemannian for classical movement. Quantum gravity should describe uniformly as quantum as classical movement. This requires a forthcoming comparison of Hilbert and pseudo-Riemannian space as well as one, already started, of quantum and classical movement.

Hilbert vs. pseudo-Riemannian space - as actual vs. potential infinity: Two oppositions are enough to represent that comparison from the viewpoint of philosophy:

- Hilbert space is 'flat', and pseudo-Riemannian space is "curved"

- Any point in Hilbert space represents a complete process, i.e. an actual infinity, and any trajectory in pseudo-Riemannian space a process in time, i.e. in development, or in other words, a potential infinity.

\section{Hilbert vs. pseudo-Riemannian space: completing the puzzle (figure 12):}

\begin{tabular}{|c|c|c|}
\hline $\begin{array}{l}\text { Oppo- } \\
\text { sition }\end{array}$ & Process in time & Actual infinity \\
\hline Curve & $\begin{array}{l}\text { Pseudo- } \\
\text { Riemannian } \\
\text { space } \\
\text { Gravity, General } \\
\text { relativity }\end{array}$ & $\begin{array}{c}\text { Banach space } \\
\text { Entanglement } \\
\text { Quantum information }\end{array}$ \\
\hline Flat & $\begin{array}{l}\text { Minkowski } \\
\text { space } \\
\text { Electromag- } \\
\text { netism } \\
\text { Special relativity }\end{array}$ & $\begin{array}{c}\text { Hilbert space } \\
\text { Electromagnetic, weak } \\
\text { and strong interaction } \\
\text { Quantum mechanics } \\
\text { The standard model }\end{array}$ \\
\hline
\end{tabular}

Figure 12
Quantum mechanics uses Hilbert space while general relativity pseudo-Riemannian space. Our objectivity is to build the bridge between those two mathematical spaces which to link those two theories.

To construct it, one should utilize the table (fig. 12), which shows those two spaces as thoroughly opposite in two features:

- curve / flat;

- process in time/ actual completeness.

Minkowski and Banach space can solve the puzzle filling up the table.

Our thesis in terms of that table (figure 13):

The thesis of this paper can be abstracted by the first row of the table above (fig. 12) as a kind of equivalence between gravity and entanglement sharing a hidden common essence, but representing it in two opposite aspects:

- as a process in time (gravity);

- as an actual completeness (entanglement).

Quantum mechanics requires that duality of process \& completeness. It roots in set theory already by means of the axiom of choice and Skolem's "paradox".

\section{Our thesis in terms of that table}

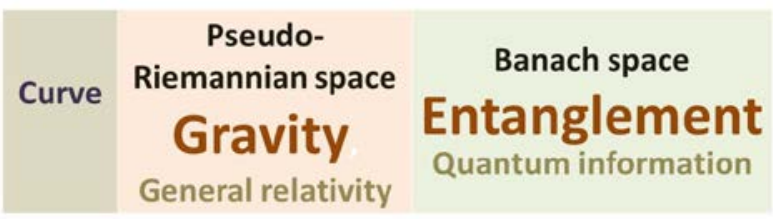

Entanglement is gravity as a complete process Gravity is entanglement as a process in time,

\section{The thesis}

Figure 13 


\section{A fundamental prejudice needs elucidation not to bar:}

The complete wholeness of any process is "more" than the same process in time, in development. Actual infinity is "more" than potential infinity. The power of continuum is "more" than the power of integers.

The objects of gravity are bigger than the objects of quantum mechanics. The bodies of our everyday world are much "bigger" than the "particles" of the quantum world, and much smaller than the universe.

Why be that prejudice an obstacle?

According to the former three statements entanglement should be intuitively "more" than gravity. However according to the latter two statements gravity should be intuitively much "bigger" than entanglement: Consequently a contradiction arises according to our intuition: Gravity should be as "less" in the first relation as much "bigger" in the second relations

An obvious, but inappropriate way out of it is to emphasis the difference between the relations:

Why is such a way out inappropriate?

The former relation links the mathematical models of entanglement and gravity, and the latter does the phenomena of gravity and entanglement. To be adequate the relations to each other, one must double both by an image of the other relation into the domain of the first one. However one can show that the "no hidden parameters" theorems forbid that. For that our way out of the contradiction must not be such a one, and:

\section{Cycling is about to be our way out of the contradiction (figure 14):}

\section{Cycling is about to be our way out of the contradiction}

Should merely glue down both ends to each other: the biggest as the most to the least as the smallest. However there is a trick: There not be anymore the two sides conformably of the "big or small" as well as of the "more or less" but only a single one like this:

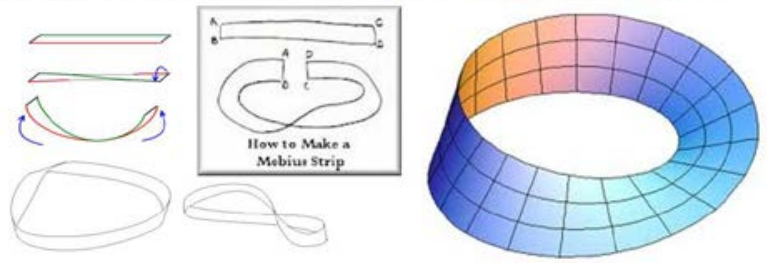

Figure 14

The cycling allows of Nicolas of Cusa's views to be used in mathematical and physical theories. It is an appropriate tool for the philosophical idea of totality or wholeness as well as the mathematical and philosophical idea of infinity to be applied and successfully used in the physical theories of quantum mechanics. It helps us to build the cherished bridge from the most biggest, the universe, to the most least, the quanta, as well as between their corresponding theories, namely general relativity and quantum mechanics. However, it means some as if metaphysical and speculative, perhaps even doubtful and dangerous ideas from philosophy and mathematics to sneak in an experimental science

like physics. This is not so. In fact, they have been in quantum mechanics for a long time, but disguised in the form of the very complicated mathematical apparatus of the contemporary physical theories.

An objective of ours is to be clearly shown the simple (but maybe inadmissible or at least, inacceptable) philosophical and mathematical fundament for them, and so their meaning and sense to be emphasized and silhouetted. Here and on the Möbius strip is utilized as the simplest possible illustration as to the cycling, as well as to the main idea of the gauge theories. This is so since the Möbius strip is one of the most elementary example of 'fiber bundle': the conception, about which is rounded as the gauge theories as the standard model. 


\section{Once again the pathway by the Möbius strip is ... (figure 15):}

\section{Once again the pathway is ...:}

$>$ from the two sides of a noncyclic strip

$>$ to the two cyclic sides of a cylinder

$>$ to a single and cyclic side of a Möbius strip

$>$ to an inseparable whole of a merely "much"

$>$ to the last one as the "second" side of the

Möbius band cyclically passing into the other

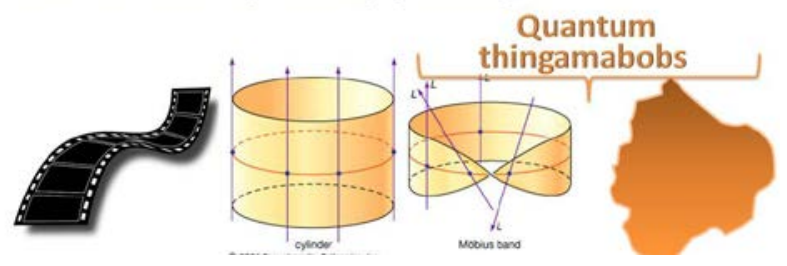

Figure 15

The Möbius strip represents well the 5, 6 , or 7 additional and cyclic pico-dimensions of string, superstring, or M-theories, too. The cyclic hyper-surfaces should be similar to the Möbius strip not to require one more dimension for its other "side". It gives us the idea of 'topological string': we can easily imagine the place, where the strip is "glued", to move gradually over its length as a wave.

That kind of theories as well as the gauge theories and the standard model share some elements of cyclicity, which is opposed to the ordinary linearity and smoothness of spacetime, though. This is inevitable if one wishes to consider together as quantum mechanics as

general relativity.

By the way, one can say that the string, superstring, or M-theories translate the discrete quantum mechanics into the language of the smooth general relativity by means of the conception of pico-"string" or cyclical additional dimensions utilizing the immanent cyclicity or wholeness of quantum mechanics. Our approach is another answer of the same epistemological tension between linearity and smoothness, on the one hand, and wholeness, cyclicity, and discreteness, on the other hand. The very, very material distinction consists of our searching of that generalizing viewpoint, from which the two extremes of the tension are equivalent, relative or invariant, while as the string, superstring, and M-theories as the gauge theories and the standard model divide them in different topoi of different dimensions (the formers) or in those of different symmetries, i.e. groups (the latters).

Holism of the East vs. linear time of the West: The edge of gluing the Möbius strip is a very special kind: It is everywhere and nowhere. We can think of it in terms of the East, together:

- as Taiji 太極 (literally "great pole"), or "Supreme Ultimate“;

- as Wuji 無極 (literally "without ridgepole") or "ultimateless; boundless; infinite“.

As a rule, the West thought torments and bars quantum mechanics: It feels good in the Chinese Yin-Yang holism. (In the West, to be everywhere and nowhere is God's property).

The "Great Pole" of cycling in terms of the axiom of choice or movement: The "Great Pole" as if "simultaneously" both (1) crawls in a roundabout way along the cycle as Taiji, and (2) comprises all the points or possible trajectories in a single and inseparable whole as Wuji

By the way, quantum mechanics itself is like a Great Pole between the West and the East: It must describe the holism of the East in the linear terms of the West, or in other words whole as time. Being people of the West, we should realize the linearity of all western science!

Physics incl. quantum mechanics is linear as all the science, too:

For example we think of movement as a universal feature of all, because of which there is need whole to be described as movement or as time. In terms of the Chinese thought, it would sound as Wiji in "terms" of Taiji, or Yin in "terms" of Yang. 
Fortunately, the very well developed mathematics of the West includes enough bridges to think of whole linearly: The most essential and important link among them is the axiom of choice:

The axiom of choice self-referentially: The choice of all the choices is to choose the choice itself, i.e. the axiom of choice, itself or in philosophical terms to choose between the West and the East. However it is a choice already made for all of us and instead of all of us, we being here (in the West) and now (in the age of the West). Consequently we doom to think whole as movement and time, i.e. linearly. The mathematical notions and conceptions can aid us in uniting whole and linearity (interpreted in physics and philosophy as movement and time), though. In particular, just this feature of mathematics determines its leading role in contemporary physics, especially quantum mechanics.

Boson - fermion distinction in terms both of whole and movement: The two version of any fermion with different spin can be explain in terms of the whole as the same being correspondingly insides and outsides the whole since the outsides of the whole has to be inside it in a sense:

A "Möbius" illustration of how a smooth trajectory can reverse the spin

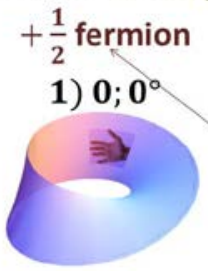

2) $\pi ; 180^{\circ}$

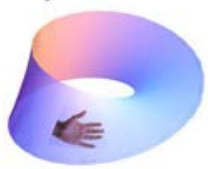

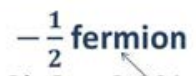

3) $2 \pi ; 360^{\circ}$

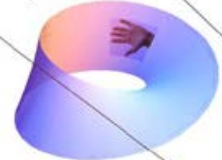

4) $3 \pi ; 540^{\circ}$

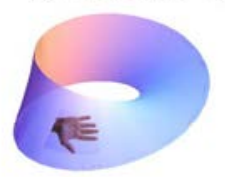

Figure 16 $+\frac{1}{2}$ fermion

5) $4 \pi ; 720^{\circ}$

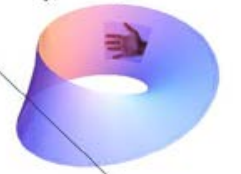

a the same fermion

"inside" "outside"

the universe
As an illustration, a fermion rotated through a full $360^{\circ}$ turns out to be its twin with reversed spin: In other words, it turns "outsides" after a $2 \pi$ rotation in a smooth trajectory passing along the half of the universe. Look at it on a Möbuis strip (figure 16):

Of course, this is a right palm, which remains right, and only its thumb is pointing down (3) instead of up (1) after a turnover. However one can equivalently think of a right glove, which is turned internal out gradually, and thus it is transformed into a left one. Analogically, the second fermion is thought as the first one but turned "internal out" like the glove above, i.e. as the same fermion.

Exactly the half of the universe between two electrons of a helium atom (fig. 17):

A helium atom can make visible the link between the most immense, the universe, and the least, a helium atom: It has only two electrons, which are the same, but their spin is opposite. One can think of them as a single electron both within and out of the universe.

Since the universe is all, its "out" should be somehow "within" it. So the second electron is as if the "image" of first one on the "mirror" of wholeness or cyclicity.

One can fancy that as a holographic image of a single electron in $\boldsymbol{d}$ dimensions for two ones in $\boldsymbol{d}+\mathbf{1}$ dimensions.

\section{Exactly the half of the universe between two electrons of a helium atom}

Here is a helium atom. Exactly the half of the universe is inserted between its two electrons which differ from each other only with reversed spin: The West thinks of the universe as the extremely immense, and of the electrons and atoms as the extremely tiny. However as quantum mechanics as Chinese thought shows that they pass into each other everywhere and always

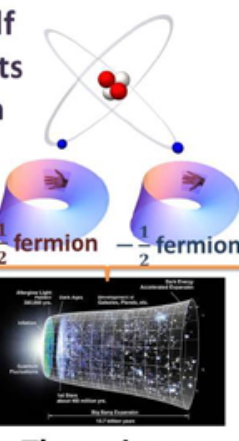

The universe 


\section{Taiji 太極 is the Chinese transition between the tiniest \& the most immense (18):}

Taiji 太極 is the Chinese transition between the tiniest and the most immense

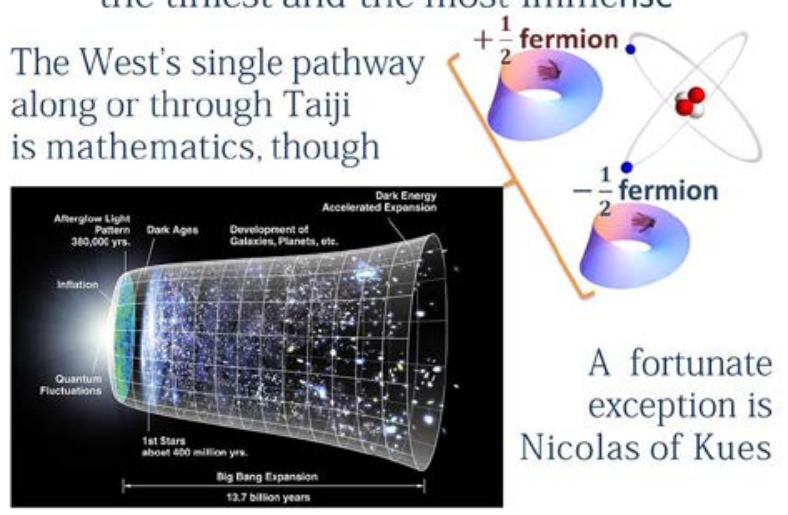

Figure 18

How on Earth is it possible? Mathematics offers the universe to be considered in two equivalent Yin - Yang aspects corresponding to quantum field (bosons) and quantum "things" (fermions): an unorderable at all set for the former, and a well-orderea space for the latters.

It is just the axiom of choice (more exactly, Scolem's "paradox") that makes them equivalent or relative. Hilbert space unites both aspects as two different (and of course, equivalent by means of it) interpretations of it: (1) as the characteristic function of a complex (or two real) quantity(es) (quantum field, bosons), and (2) as a vector (or a square integrable function).

Taiji 太極 in the language of mathematics (figure 19, 20 \& 21):

Taiji aids us to understand the universe in its two "hypostases":

- as a mere and inseparable "much", which is natural to be called "Yin" in the Chinese tradition, and

- as a well-ordered "many" clearly recalling for the Chinese "Yang".

So seen, Yin and Yang are rather dividing from each other, and Taiji remains that "Great Pole", from which their unity can be yet watched. It conserves the idea of their equivalence, relativity or even invariance despite of their clearly visible oppositeness.

Taiji 太極 in the language of mathematics

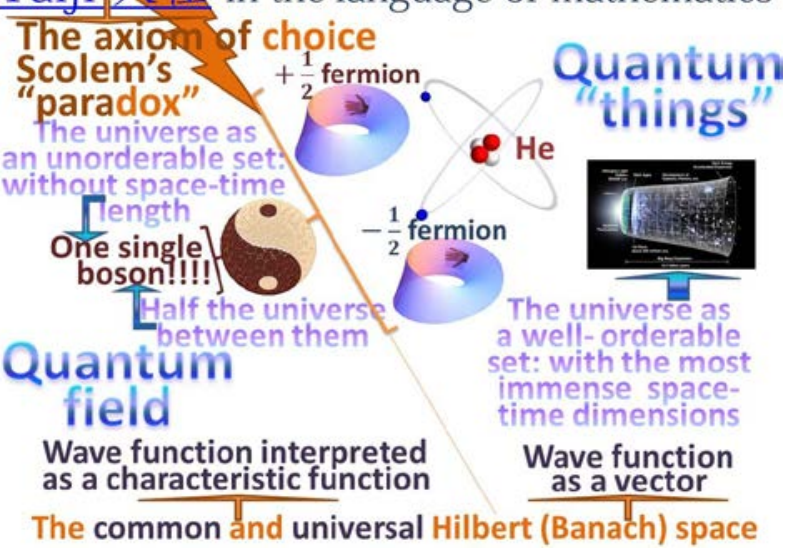

Figure 20

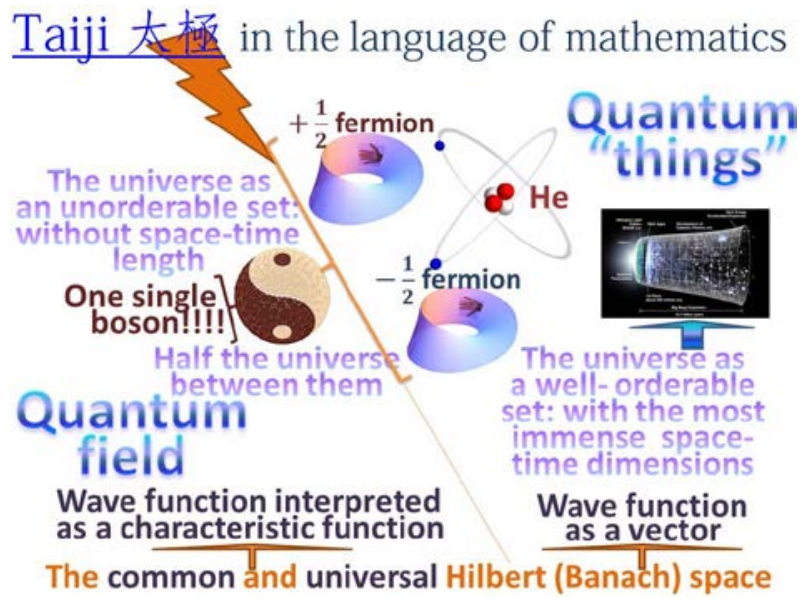

Figure 19

Taiji interpreted this way is already easily translatable as the axiom of choice in the language of mathematics (figure 20). However the Chinese thought of Taiji has yet contented important instructions rather uninvestigated by the European science. They are known for it as Skolem's "paradox" (without quotation marks).

One ought to put the quotation marks for the context of Taiji since it is not paradoxical in the Chinese understanding, but only for the European rather misunderstanding than understanding. Quantum mechanics, an experimental science, is what imposed on the West to

enter the relatıvity of intınıty not as a paradox. 
In particular it involves a new conception of calculation as well of the mathematical model for it, namely 'quantum computer' (figure 21), which, besides, understands all the physical processes as computational ones. If mankind ever manages to construct the machine, which to realize the mathematical model of quantum computer, then its computations would generate the physical being from nothing ("ex nihilo"), i.e. from a pure computation though quantum.

Quantum mechanics is featured by the convergence even coincidence of nature and technics (an idea of Heidegger's for the Greeks).

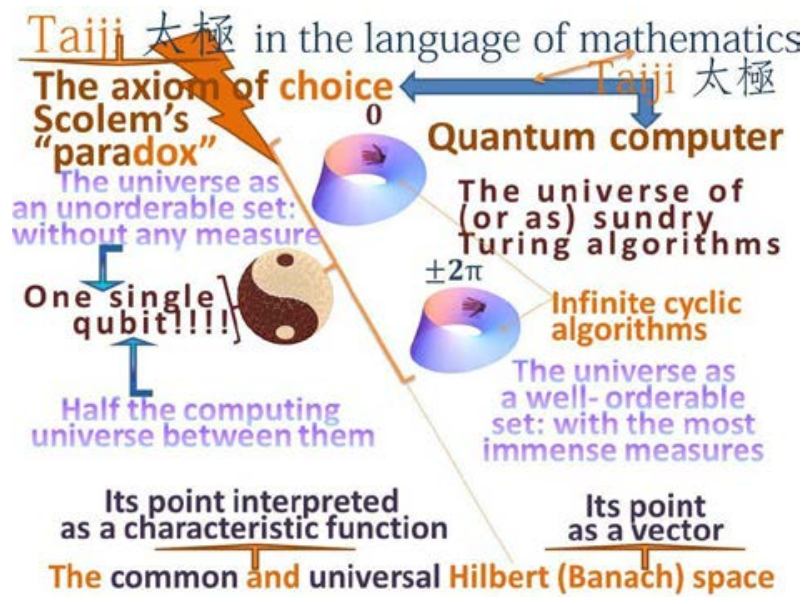

Figure 21

Wuji 無極 as the Kochen-Specker theorem (figure 22):

If the axiom of choice will be the

Wuji 無極 as the Kochen-Specker theorem The axiom of choice Taiji 太極

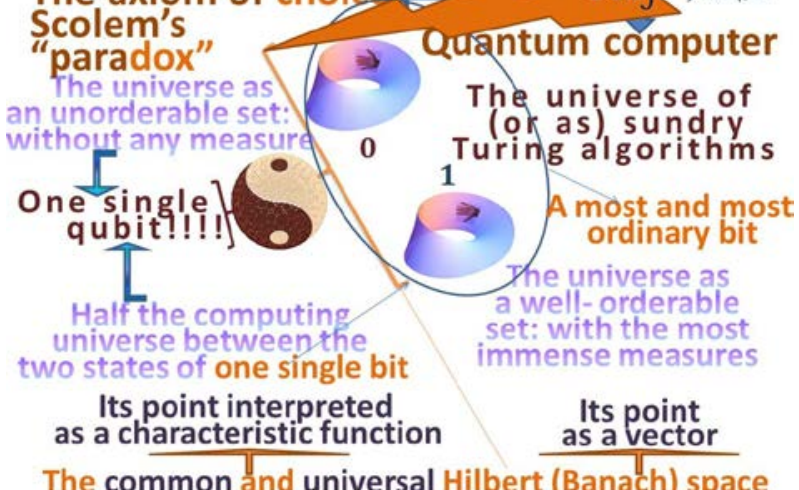

Figure 22 translation of "Taiji" into the language of mathematics, then which should the translation of its counterpart, "Wuji” be?

The Kochen - Specker theorem in quantum mechanics is opposed to the axiom of choice as Wuji to Taiji. Its meaning is no quantum whole, e.g. no qubit, can be divided into two (consequently, or more) disjunctive parts, or in other words, no part of its can be chosen.

Thus the axiom of choice states that a part can always be chosen, and the Kochen Specker theorem says that a part can never be chosen. A third option remains between them:

that some parts can be sometimes chosen. Consequently, the axiom of choice for Taiji, and the Kochen - Specker theorem for Wuji are two poles of one single whole like the quantum one, which cannot be thoroughly reduced to its two poles.

Given the axiom of choice as above (figure $20 \& 21$ ), the Kochen - Specker theorem can yet translated in its terms as Skolem's "paradox", or as the relativity of infinity (as well as Skolem's "relativity of the notion of "set"), or as the equivalence of positive integers and any set, or as an invariance of the discrete and continuous (smooth) in quantum mechanics and general relativity.

Moreover, one can think of the true "Big Bang" as such a third between the two poles of the purely mathematical, on one hand, and the purely physical, on the other hand, or in other words, between the one hypostasis of the being and universe as a mere and inseparable "much" (for the Kochen - Specker theorem and other "no-hidden-variables" theorems) and the other one, that of the actually divided "many" well-ordered in space-time. So the "Big Bang" is necessarily required by the Kochen - Specker theorem, or said at all, by the laws of nature, including as those of physics as those of mathematics. One can emphasize: 
The “Big Bang” needn't another cause beside the mathematical laws of nature. Consequently, no moment in time (e.g. 14-15 billion years ago) when it has happened since the laws act always. It happens constantly like them, too, and the "Big Bang" is only an alleged projection of its constant happening into a definite moment in time:

Utilizing cyclicity, one can show unrigorously that only two constants, those of Plank, and of the light speed in vacuum always generates an effect integrable and projectable as the hypothetical "Big Bang”. Besides, that effect can be reconfirmed as to the 3D Euclidean space by means of the Poincaré conjecture already proved by Perelman.

Indeed, the ratio of the Planck constant (h) and the light speed in vacuum (c) represents the length $(x)$ per a unit of cycle $(y)$ :

$$
y=\frac{\hbar}{c} \leqq x \cdot m
$$

Equation 7

Here " $m$ " means not directly the quantity of mass (or energy), but rather the curvature caused by this mass (energy), i.e. the measure of deformation of a circle in a plane of pseudo-Riemannian space. Consequently, $y$ is the quant of a cycle, i.e. the minimally possible length of a cycle permitted by the laws of nature. Thus a cycle in space (as any quantity in quantum mechanics) should be both a discrete quantity, i.e. some number of the quant, $y$, and a continuous (in fact, even smooth) quantity in time as the speed is defined and finite. However, this can take place only under two conditions: (1) the length of a cycle is infinite; (2) the axiom of choice is valid (any quant of that cycle can be isolated). Consequently the continuous space, which is equivalent with that infinite cycle, must be necessarily infinite, too. Since the space being namely continuous is created with the light speed in vacuum, the contradiction of its infiniteness and its finiteness in any moment in time can be equated only by the "Big Bang", which, by the way, will seem more and more stronger with more and more longer periods of time as an integral in time projected in one beginning of the beginnings constant as a moment in time - that of the universe.

In other words, the "Big Bang" is only an epiphenomenon: not a real phenomenon, but such a one, which arises on the screen of our cognition in order to explain the too strange quantum by our up-to-date background knowledge. It is not more than an image of the quantum equivalence of the discrete and continuous (smooth) as to a special physical quantity, that of distance.

The above consideration refers only to a one-dimensional space while the physical space is three-dimensional. The conjecture that it can be merely generalized to any finitedimensional space is not correct. There are numbers of dimension, for which this is not true. Fortunately, Perelman's proof frees us from the troubles as to the physically meaningful case of three dimensions. Indeed the 3-sphere can be seen as a three-dimensional equivalent of a onedimensional cycle discussed above, and the 3D Euclidean space is a particular case of an open 3D manifold. This allows of the Poincaré conjecture to be applied in our case.

Some questions remain open: first of all, that for the relation between the topological Poincaré conjecture and the set-theory axiom of choice. The second one refers to the axiom of mathematical induction in Peano arithmetic. It (as well as Peano arithmetic for that) 
admits two interpretations: the linear (usual) one and the cyclic one. Consequently if any of them is available the other is necessarily available, too. The problem is whether the last statement is an arithmetical equivalent or fundament of the equivalence of the discrete and continuous in quantum mechanics requiring in turn the axiom of choice. Their investigation would digress from our objectivities. However one should emphasize that the fundamental mathematical questions are closely connected and often entangled with those of physics and quantum mechanics as the following is going to show in particular:

The informational and mathematical structure of electromagnetic wave: Being a physical process, it realizes the well-ordering of space-time physically. Consequently, it must be the boundary between the well-ordering of space-time, on the one hand, and the unorderable, required by the "no hidden variables" theorems in quantum mechanics, on the other hand. Being that boundary, it acts as a mediator between those two domains: This predefines for it a certain mathematical and logical structure, which represents the elementary structure of a choice, i.e. one bit as an informational unit or a graph node. The elementary choice as an information bit can be thought as a cell (e.g. of a Turing machine), on which can be written one of two disjunctive options (e.g. either 0 or 1). Consequently the light wave is what writes in the empty cell as well as both the written and the cell. One should try to see its primary and mathematical structure as such an informational bit by means of electrical (mono-)poles and magnetic dipoles (by the way, it explains why there are no magnetic poles in nature):

- The electric pole is as an empty cell.

- The magnetic dipole is as two options for the record.

- The choice between the two options turns the chosen option in a new, following empty cell, i.e. into an electric pole.

- The cycle begins again from the beginning. The cycle of such a discrete choice between two options is continuous, though.

One can see how two of Maxwell's equations (accepting that they all are four) describe the above elementary informational structure: any volume can content an electric pole; no volume can content a magnetic pole, but it can content a magnetic dipole.

Dirac's spinors keep this primary and informational structure generalizing it from a bit to a qubit:

More about Dirac's spinors: One can think of them both ways: as two electromagnetic waves; as the complex (=quantum) generalization of electromagnetic wave. The latter is going to show us the original Dirac theory. However the former is much more instructive and useful for our objectives: It is going to show us the connection and unity of gravity and electromagnetism, and hence then the links of gravity and quantum theory by the mediation of electromagnetism.

The more general consideration is the former: It admits more degrees of freedom as an arbitrary angle between the two axes of the electromagnetic waves since that angle is fixed as $90^{\circ}$ in the latter case. It and the corresponding degree of freedom appear only between spinors for the quantum uncertainty (or for the qubit generalization), which is an additional degree of freedom. Consequently it cannot be available between the components of the classical electromagnetic wave described by Maxwell's equations. 
That angle can be interpreted as a curvature of the space, in which the electromagnetic wave is spread or as caused by gravity. It appears for the entanglement of the electromagnetic wave with all the rest and allows of thinking about gravity as entanglement.

The space-time, in which an electromagnetic wave is spread, and the space, in which a mass point moves share a common formalism, that of pseudo-Riemannian space (in particular, Minkowski space) and this permits mass and energy to be equated as Einstein did. However a slight distinction can be yet made between the two cases:

The space-time of electromagnetic wave, i.e. the former case: The angle is internal in a qubit, e.g. as an angle between two great circles of a sphere, which are enough to describe it. One can say that thus space-time appears this way as a well-ordering and "from nothing", i.e. from the unorderable in principle. The curvature of space-time can be seen as an arbitrary spin (i.e. not only multiply of $1 / 2$ ) of that qubit.

Consequently the superluminal domain of special relativity can be identified as the pseudo-Riemannian space of general relativity through or by an intermediate stage of "pure" (or quantum) probability, through or by which that probability can undergo the direct influence of other probabilities, which change and deform it by entanglement. After that deformation, it "returns" (as if returns) in a subluminal domain of well-ordering, which is already deformed in turn as pseudo-Riemannian space. That deformation is caused by entanglement, but it is seen in space-time as its deformation or as some "force", that of gravity. Such is its mechanism, a quantum one in essence, which is the cause not to be able to be quantized repeatedly.

Utilizing the analogy with 'potential infinity' as a process of making space-time by any electromagnetic wave, one can say of "potential space-time” in that former case.

The space-time of a mass point, i.e. the latter case: The angle is external, between tangential lines in two points of a space-time curve, i.e. a world line. The space-time is already ready, grandfathered, made before that by the light (electromagnetic wave).

The construction, which is typical for special and general relativity, of the exchange of light signals between reference frames or "observers" should be slightly reinterpreted: In fact, those light signals are what make space-time, which can be already grandfathered for reference frames or observers. Of course, the space-time can be (and almost always is) grandfathered for the light signals, too, for other light signals before them. However the question, whether the former and the latter light signals are the same or different, has not an unambiguous answer. The light is (or seems as) different only in the 3D space, which is the space of our perception, but not in the space-time of special and general relativity since the space-time distance between any two light signals is always zero.

Utilizing the analogy with 'actual infinity' as a completed process of a grandfathered space-time already made somehow, one can say of “actual space-time” in that latter case.

The transition between the space-times of electromagnetic wave and a mass point: It is a boundary transition, but in the opposite direction: from the boundary to the transition. The physical interpretation of that boundary transition is well-known from special relativity: It would require infinite energy in the "right direction" and consequently the corresponding, also infinite decrease of energy in the opposite direction for a mass point. In fact, the transition is between entities without a nonzero mass at rest like the photons and particles having a nonzero mass at rest: Mass at rest and space-time distance can mutually annihilate (transform). 
Though the transition is physically forbidden to a mass point as the necessary energy is infinite, mutual metamorphoses between photons and particles with some nonzero mass at rest, e.g. between a photon and the pair of an electron and a positron, is absolutely possible, plausible and often observed. That as if quantum transition obeys energy conservation and Einstein's famous formula, $E=m c^{2}$ (here " $m$ " is the relativistic mass rather than the mass at rest), after which the energy is shared between the kinetic energy for the movement of the particle and its mass at rest.

In Minkowski space, i.e. in the mathematical formalism of special relativity, that physically possible, leap-like transition is a mapping between the light cone and some subset of its internality.

However all this is not enough in the present context: One should add a quantum comprehension and the corresponding interpretation as the invariance to the axiom of choice and to the mathematical and physical. Then the transition can be thought as a micro-local consideration of a point of space-time. Seen by that "microscope", the point seems like (and in fact, it is) a qubit admitting a decomposition to two "spinors" (two great circles, which can be arbitrarily angled in our consideration unlike the original spinors, which are always orthogonal) as above. Consequently the microscope can observe a space-time point in the process of its generation as a complicated structure. Only when it is completed and "ready for use" ("prêt-àporter"), its internal properties are transformed thoroughly into external ones, and the qubit becomes a space-time point. This course of thought resembles the gauge theories which as if place all Hilbert space and its automorphisms and symmetries within any space-time point. By the way, a qubit is equivalent to Hilbert space if the axiom of choice is given an infinite number of times. Thus our approach is within the gauge frame.

One has to understand the essence of how a mass at rest can appear after a leap from a potential to an actual space-time point or vanish in the opposite direction. However the answer will be in principle, and not why the mass at rest of one or another elementary particle is a given value. There exists a cycle of mutual transformation between mass $(m)$, energy $(E)$, time $(t)$, and quantum information $(Q)$, which is regulated by the four most fundamental constants: the Plank one $(h)$, the light speed one $(c)$, the gravitational one $(G)$, and the Boltzmann one $(k)$.

\section{Short conclusions:} entanglement:

A few principles seem to be able to underlie the unification of gravity and

1. Actual infinity should be considered as a physical and experimental subject in quantum mechanics and information and thus in quantum gravity. It is the "least common denominator", under which general relativity and quantum mechanics can be unified.

2. Cyclicity: It means for the system as a whole and any element of it to allow of their identification as a cyclic return of the system into itself obeying the law of conservation of action (or the quantity of information), which generalizes conservation of energy for that return.

3. Quantum invariance: It requires the identification of the coherent state of a quantum system before measurement with the well-ordered set of measurements of this system and requires the mathematically corresponding invariance to the availability or the absence of the axiom of choice. 


\section{Literature:}

Jacobson, T. 1995. Thermodynamics of Spacetime: The Einstein Equation of State. - Physical Review Letters, Vol. 75, Issue (August 14, 1995) pp. 1260-1263.

Lee, Jae-Weon, Hyeong-Chan Kim, Jungjai Lee. 2010. Gravity as Quantum Entanglement Force. - http://arxiv.org/abs/1002.4568.

Maldacena, J. 2011. The gauge/ gravity duality. - http://arxiv.org/abs/1106.6073.

van Raamsdonk, M. 2010. Comments on quantum gravity and entanglement. http://arxiv.org/abs/0907.2939.

Perelman, G. 2002. The entropy formula for the Ricci flow and its geometric applications. http://arxiv.org/abs/math.DG/0211159.

Perelman, G. 2003. Ricci flow with surgery on three-manifolds. http://arxiv.org/abs/math.DG/0303109.

Perelman, G. 2003. Finite extinction time for the solutions to the Ricci flow on certain threemanifolds. - http://arxiv.org/abs/math.DG/0307245.

Verlinde, E. 2009. On the Origin of Gravity. - http://arxiv.org/abs/1001.0785 